\title{
Aging-induced dielectric relaxation in barium titanate ceramics
}

Mohamad M. Ahmad, Koji Yamada, Paul Meuffels, and Rainer Waser

Citation: Appl. Phys. Lett. 90, 112902 (2007); doi: 10.1063/1.2713178

View online: https://doi.org/10.1063/1.2713178

View Table of Contents: http://aip.scitation.org/toc/apl/90/11

Published by the American Institute of Physics

\section{Articles you may be interested in}

Dielectric properties of fine-grained barium titanate ceramics Journal of Applied Physics 58, 1619 (1985); 10.1063/1.336051

Migration and redistribution of oxygen vacancy in barium titanate ceramics

Applied Physics Letters 89, 071916 (2006); 10.1063/1.2337097

Domain wall clamping in ferroelectrics by orientation of defects Journal of Applied Physics 73, 3454 (1993); 10.1063/1.352948

The effect of grain and particle size on the microwave properties of barium titanate $\left(\mathrm{BaTiO}_{3}\right)$ Journal of Applied Physics 83, 3288 (1998); 10.1063/1.367097

Aging of the Properties of Barium Titanate and Related Ferroelectric Ceramics

The Journal of the Acoustical Society of America 27, 73 (1955); 10.1121/1.1907500

Internal bias in acceptor-doped $\mathrm{BaTiO}_{3}$ ceramics: Numerical evaluation of increase and decrease

Journal of Applied Physics 68, 4220 (1990); 10.1063/1.346212

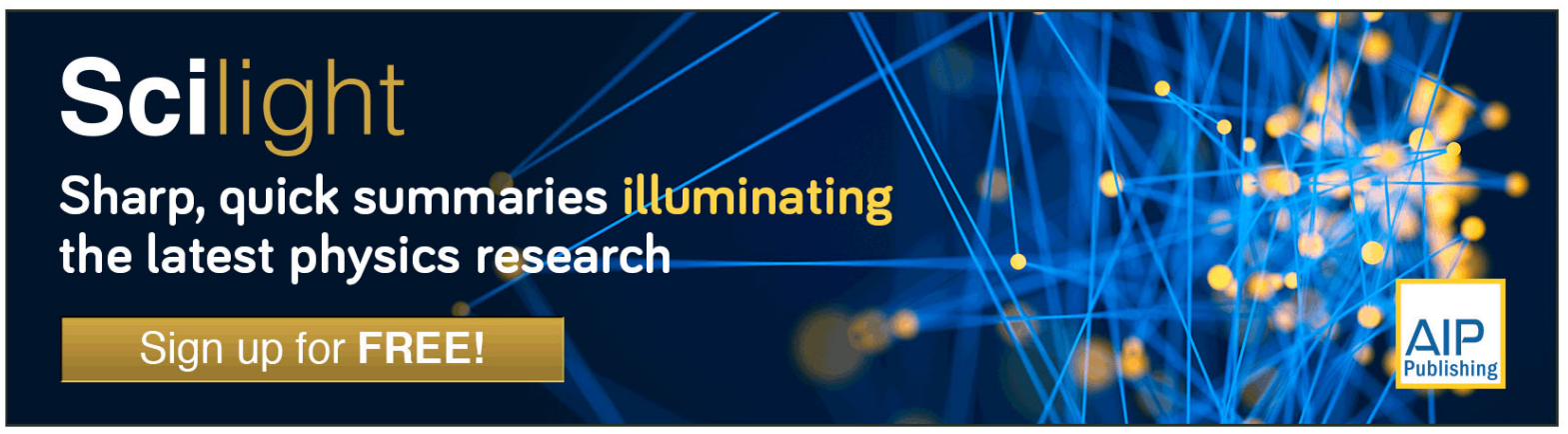




\title{
Aging-induced dielectric relaxation in barium titanate ceramics
}

\author{
Mohamad M. Ahmad ${ }^{\text {a) }}$ and Koji Yamada \\ Department of Applied Molecular Chemistry, College of Industrial Technology, Nihon University, \\ Narashino, 275-8575 Chiba, Japan \\ Paul Meuffels and Rainer Waser \\ Institute of Solid State Research, Research Center Juelich, D-52425 Juelich, Germany
}

(Received 10 January 2007; accepted 8 February 2007; published online 12 March 2007)

\begin{abstract}
In this letter the authors report on the aging effect on the phase transitions and dielectric properties of $1 \mathrm{~mol} \% \mathrm{Mn}$-doped $\mathrm{BaTiO}_{3}$ ceramics. Aging the ceramics in the ferroelectric state stimulates a resistance for the orthorhombic-tetragonal and tetragonal-cubic phase transitions, which leads to stabilization of the tetragonal phase over a wider temperature range. The dielectric constant of the aged sample shows dispersion, associated with a dielectric loss relaxation peak near the $100 \mathrm{kHz}$ frequency region, in contrast to the unaged one. The present results are discussed in light of the existing models for ferroelectric domain stabilization. (C) 2007 American Institute of Physics. [DOI: $10.1063 / 1.2713178$ ]
\end{abstract}

Ferroelectric materials with perovskite structure have found wide applications in the electronic industry for the fabrication of multilayer ceramic capacitors and ferroelectric memories. ${ }^{1}$ However, in these materials a wide variety of point defects can be formed, which play a vital role in tailoring ferroelectrics to their many important applications. Accordingly, aging phenomena, a time-dependent changing of material properties, occur in ferroelectrics and the reliability and lifetime of electronic components and devices based on them are greatly reduced. ${ }^{2}$ In this regard, the role of oxygen vacancies is often the proposed culprits in various reliability limiting processes in ferroelectrics. Therefore, intensive investigations on the complex motion and the relaxation processes of oxygen vacancies are required to solve the degradation issue in ferroelectrics based devices.

Aging effect in ferroelectrics usually manifests itself by a gradual decrease of the small signal dielectric and piezoelectric parameters, as well as the appearance of a shift of the large signal polarization-electric field hysteresis loop along the axis of electric field. ${ }^{3-8}$ It is generally accepted that aging effect is originated from a gradual stabilization of the existing ferroelectric domains by defects. ${ }^{3-5}$ However, various theoretical models, suggesting boundary ${ }^{6,7}$ or volume ${ }^{3-5}$ effects as origins of the ferroelectric domains stabilization, have been proposed in the literature. The aging behavior of the small signal dielectric properties in bulk acceptor-doped ferroelectric materials is attributed to a reduction of the domain wall contributions to the dielectric properties. This reduction was suggested to come from the stabilization of the domain walls in their positions either by diffusion of oxygen vacancies into and pinning of domain walls (boundary effect) $)^{6,7}$ or by clamping of domain walls (volume effect). 3,5 In their model, Robels and Arlt explained the clamping of domain walls as a result of time-dependent orientation of defect dipoles (formed by oxygen vacancies and defect centers in Ti positions) parallel to the polarization within the ferroelectric domains. ${ }^{5}$ The aligned defect dipoles, associated

\footnotetext{
${ }^{a}$ On leave from Department of Science and Math, Faculty of Education, Assiut University Branch in The New Valley, Egypt; electronic mail: m5ahmad@cit.nihon-u.ac.jp
}

with defect dipole polarization, produce electric internal field, which stabilizes the ferroelectric domains. In the equilibrated aged ferroelectric state, when applying small signal ac field, as in the dielectric measurements, the domain wall motion encounters a resistance from the defect polarization that keeps its orientation. ${ }^{3-5}$ This leads to a reduction of domain wall mobility compared to the unaged state, and hence a decrease in the dielectric properties. In this letter, based on dielectric measurements, we report interesting results regarding the stabilization of the ferroelectric tetragonal phase and the aging-induced low frequency dielectric relaxation behavior in $1 \mathrm{~mol} \% \mathrm{Mn}$-doped $\mathrm{BaTiO}_{3}$ (BTO-1Mn hereafter) ceramics.

In BTO-1Mn samples, $\mathrm{Mn}$ was added as acceptor dopant, where its valence state is $3+$ after sintering at high temperatures in air. ${ }^{9}$ Oxygen vacancies are simultaneously created by charge compensation. Before the dielectric measurements, BTO-1Mn sample was cooled through its cubictetragonal transition temperature and followed by aging at $90{ }^{\circ} \mathrm{C}$ for five weeks. Dielectric measurements of the aged sample were performed in the $-13-200{ }^{\circ} \mathrm{C}$ temperature range at a heating rate of $0.2{ }^{\circ} \mathrm{C} / \mathrm{min}$. The sample is then refreshed at $250{ }^{\circ} \mathrm{C}$ for $5 \mathrm{~h}$ followed by fast cooling at a rate of $15-20^{\circ} \mathrm{C} / \mathrm{min}$. Dielectric measurements of the refreshed sample were performed under the same conditions as the aged one. Dielectric measurements were carried out in the frequency range of $50 \mathrm{~Hz}-5 \mathrm{MHz}$ using HIOKI 3532 meter.

Figures 1(a) and 1(b) present the temperature dependence of the real part of the dielectric constant $\varepsilon^{\prime}$ at selected frequencies in the vicinity of the tetragonal-cubic and the orthorhombic-tetragonal phase transitions, respectively, for the aged and refreshed samples. Interesting features can be observed in this figure; (i) in Fig. 1(a) one notices that the temperature of the tetragonal-cubic transition of the aged sample is shifted about $1.5^{\circ} \mathrm{C}$ higher than the refreshed sample, whereas in Fig. 1(b) the orthorhombic-tetragonal transition temperature of the aged sample is shifted about $4{ }^{\circ} \mathrm{C}$ lower than the refreshed sample. These results indicate that aging BTO-1Mn in the ferroelectric state stabilizes the tetragonal ferroelectric phase by not only shifting the high temperature transition (reported previously by Sun et al.) ${ }^{10}$ 

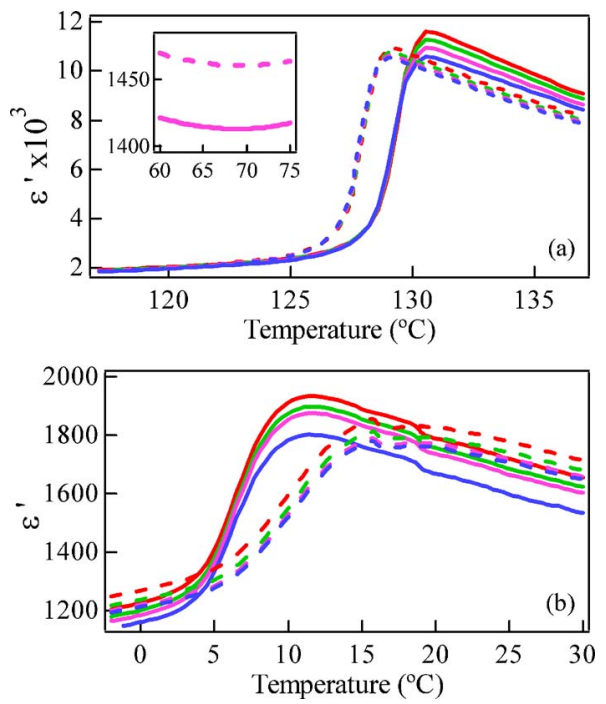

FIG. 1. (Color online) Temperature dependence of $\varepsilon^{\prime}$ for the aged (solid lines) and refreshed (broken lines) samples (at 0.096, 0.96, 9.65, and $96.5 \mathrm{kHz}$ from top to bottom) in the vicinity of (a) the tetragonal-cubic and (b) orthorhombic-tetragonal phase transitions. The inset in (a) shows the temperature dependence of $\varepsilon^{\prime}$ in the tetragonal phase at $9.65 \mathrm{kHz}$.

but also by shifting the room temperature phase transition to lower temperature, which was not reported previously. (ii) The values of $\varepsilon^{\prime}$ for the aged sample in the tetragonal phase are reduced compared to the unaged one [inset of Fig. 1(a)]. (iii) The values of $\varepsilon^{\prime}$ for the aged sample at the transition peaks and in the high temperature paraelectric phase are higher than those of the refreshed one. Dielectric dispersion of the aged sample, where its $\varepsilon^{\prime}$ values decrease with increasing frequency, is also observed in the paraelectric phase.

The results in Fig. 1 can be explained by the volume effect model of ferroelectric domain stabilization, ${ }^{5}$ supported by the microscopic picture produced by Zhang and Ren for the aging in ferroelectrics. ${ }^{3}$ For single-domain Mn-doped $\mathrm{BaTiO}_{3}$ single crystal, Zhang and Ren presented a clear evidence that aging of polarization hysteresis loop stems only from volume effect due to the stabilization of ferroelectric domains by defect dipoles. ${ }^{3}$ According to these models, immediately after cooling the fresh sample through its ferroelectric phase the crystal symmetry changes to the polar tetragonal symmetry while oxygen vacancies surrounding a central $\mathrm{Mn}^{3+}$ defect in the distorted oxygen octahedron retain their cubic symmetry, which represents unstable configuration. During aging (which requires time), oxygen vacancies diffuse and rearrange to create defect dipoles with $\mathrm{Mn}^{3+}$ defects, whose symmetry follows the crystal symmetry. This stable configuration stabilizes the ferroelectric domains in their current positions and exhibits a resistance to both the room and high temperature transitions, leading to expansion of the tetragonal ferroelectric range. It is noteworthy mentioning that theoretical calculations by Park and Chadi on $\mathrm{PbTiO}_{3}$ indicated that oxygen vacancies in the Ti-O-Ti chains along the polarization axis are more stable than those in Ti-O-Ti planes normal to the axis by $0.3 \mathrm{eV} .{ }^{11}$ Consequently, oxygen vacancies in the $a b$ plane have tendency to migrate to the more favorable sites in the Ti-O-Ti chains along $c$ axis in the course of aging.

The reduction of $\varepsilon^{\prime}$ after aging can be simply explained as a result of domain walls stabilization, as discussed above. Additionally, the large values of $\varepsilon^{\prime}$ at the transition peaks

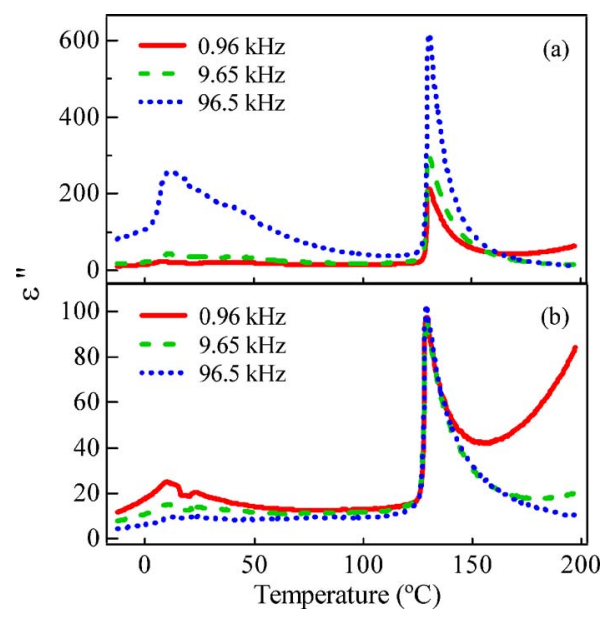

FIG. 2. (Color online) Temperature dependence of $\varepsilon^{\prime \prime}$ at selected frequencies for the (a) aged and (b) refreshed samples.

and in the paraelectric phase of the aged sample compared to the refreshed one may be a result of some memory effect. Immediately after passing the tetragonal-cubic transition during heating, the crystal symmetry changes to cubic by diffusionless transformation, where no more ferroelectric domains exit. On the other hand, defect dipoles created during aging cannot follow the crystal symmetry, and they retain their tetragonal symmetry. ${ }^{3,10}$ These dipoles can rotate easily with the frequency of the applied ac field resulting in an enhancement of $\varepsilon^{\prime}$. After some time in the paraelectric state, however, the sample is deaged and oxygen vacancies redistribute between the equivalent six sites around the defect center in the cubic lattice.

The temperature dependence of the imaginary part of the dielectric constant, $\varepsilon^{\prime \prime}$, at different frequencies is shown in Figs. 2(a) and 2(b) for the aged and refreshed samples, respectively. Again, the stabilization of the tetragonal ferroelectric phase is manifested by the shifts in the peak temperature of the room temperature and the high temperature phase transitions. Interestingly, we notice in Fig. 2 that the value of $\varepsilon^{\prime \prime}$ at the peaks of the aged sample, at low frequencies, is roughly three times larger than the refreshed sample. This high value of $\varepsilon^{\prime \prime}$ in the aged sample is clearly related to the diffusion of oxygen vacancies during aging process. Moreover, anomalous increase in $\varepsilon^{\prime \prime}$ of the aged sample is observed at low temperatures and high frequencies.

For exploring the origin of the high frequency anomaly in $\varepsilon^{\prime \prime}$, we present the frequency dependence of $\varepsilon^{\prime}$ for the aged and refreshed samples in Figs. 3(a) and 3(b), respectively. It is well known that $\mathrm{BaTiO}_{3}$, both in the polycrystalline ceramic or polydomain single crystal forms, exhibits a large dielectric relaxation in the low gigahertz frequency region. ${ }^{12}$ It was urged that the dielectric dispersion is a result of a relaxation of domain walls that exist in ferroelectric materials. ${ }^{13}$ For the refreshed sample, Fig. 3(b), no dielectric dispersion was observed in the frequency range studied. On the other hand, the aged sample reveals a large dielectric dispersion at low temperatures for frequencies $>100 \mathrm{kHz}$. Clearly, this dispersion occurs at frequencies much lower than the gigahertz regime. Additionally, since the refreshed sample did not show dispersion in the same frequency range, it could be concluded that the observed dielectric dispersion in the aged sample is not due to domain walls relaxation. Therefore, the dielectric dispersion in the aged sample could 


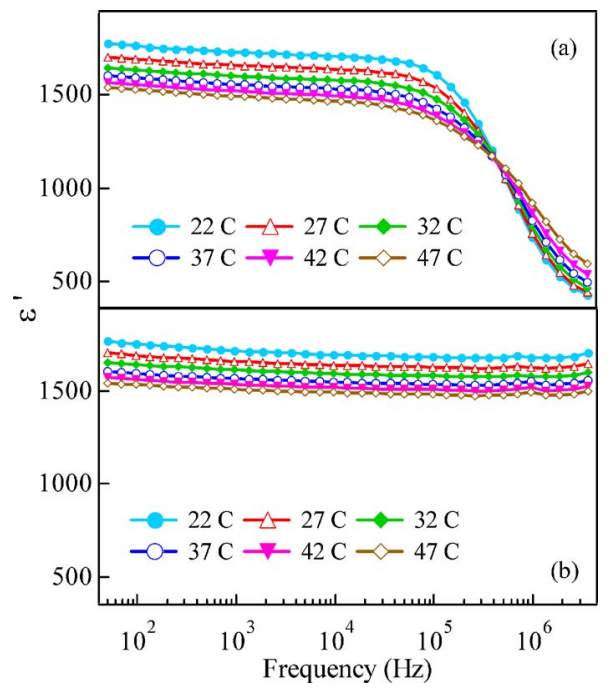

FIG. 3. (Color online) Frequency dependence of $\varepsilon^{\prime}$ at selected temperatures for the (a) aged and (b) refreshed samples.

be explained as a result of the diffusion and redistribution of oxygen vacancies during aging, which is the only difference between the refreshed and aged samples. Figure 4 shows the frequency dependence of $\varepsilon^{\prime \prime}$ at low temperatures for the aged sample, where a clear dielectric loss peak is observed at high frequencies, representing dielectric relaxation phenomena. The loss peak shifts to higher frequencies with increasing temperature, indicating a thermally activated relaxation process. The relaxation time $\tau$ is extracted from the peak maximum at different temperatures. The temperature dependence of $\tau$ can be represented by the Arrhenius relation: $\tau=\tau_{0} \exp (E / k T)$, where $k$ is Boltzmann constant, $\tau_{0}$ the preexponential factor, and $E$ the activation energy. The obtained value of $\tau_{0}=5.2 \times 10^{-11} \mathrm{~s}$ agrees with that obtained for oxygen vacancies relaxation in $\mathrm{BaTiO}_{3}$ ceramics studied by mechanical loss. ${ }^{14}$ However, the value of the activation energy $E=0.22 \pm 0.01 \mathrm{eV}$ is smaller than that proposed for oxygen vacancy diffusion process in the range of $0.4-0.68 \mathrm{eV} .{ }^{15}$ However, the low value of $E$ in the present study may be due to the short-range diffusion and rearrangement of oxygen vacancies in the vicinity of $\mathrm{Mn}^{3+}$ defects within the distorted oxygen octahedron for the formation of defect dipoles.

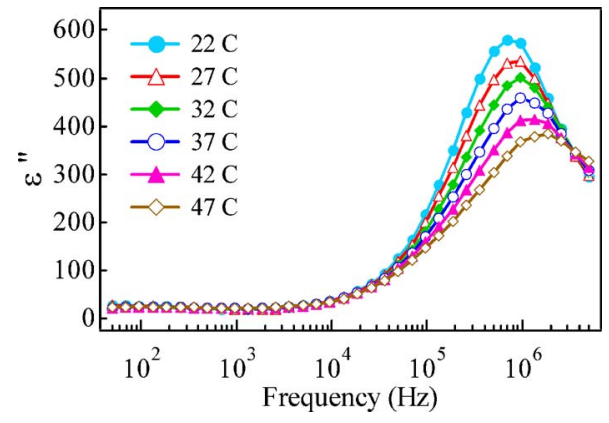

FIG. 4. (Color online) Frequency dependence of $\varepsilon^{\prime \prime}$ for the aged sample at different temperatures.

In summary, we have shown that aging BTO-1Mn ceramics in the ferroelectric state affects both the room temperature and high temperature ferroelectric phase transitions so that the tetragonal ferroelectric phase is extended over a wider temperature range, due to the stabilization of the ferroelectric domains by the reorientation and alignment of the defect dipole polarization along the polarization axis of the lattice. The diffusion of oxygen vacancies during aging induces a dielectric dispersion associated with a dielectric loss relaxation peak. The dielectric dispersion phenomena could be attributed to the relaxation of oxygen vacancies in the aged sample.

${ }^{1}$ M. E. Lines and A. M. Glass, Principles and Applications of Ferroelectrics and Related Materials (Clarendon, Oxford, 1977).

${ }^{2}$ R. Waser, T. Baiatu, and K. H. Hardtl, J. Am. Ceram. Soc. 73, 1645 (1990).

${ }^{3}$ L. Zhang and X. Ren, Phys. Rev. B 73, 094121 (2006).

${ }^{4}$ P. V. Lambeck and G. H. Jonker, J. Phys. Chem. Solids 47, 453 (1986).

${ }^{5}$ U. Robels and G. Arlt, J. Appl. Phys. 73, 3454 (1993).

${ }^{6}$ V. S. Postnikov, V. S. Pavlov, and S. K. Turkov, J. Phys. Chem. Solids 31, 1785 (1970).

${ }^{7}$ K. Carl and K. H. Hardlt, Ferroelectrics 17, 473 (1978).

${ }^{8}$ W. A. Schulze and K. Ogino, Ferroelectrics 87, 361 (1988).

${ }^{9}$ A. Kirianov, N. Ozaki, H. Ohsato, N. Kohzu, and H. Kishi, Jpn. J. Appl. Phys., Part 1 40, 5619 (2001).

${ }^{10}$ D. Sun, X. Ren, and K. Otsuka, Appl. Phys. Lett. 87, 142903 (2005).

${ }^{11}$ C. H. Park and D. J. Chadi, Phys. Rev. B 57, R13961 (1998).

${ }^{12}$ A. Von Hippel, Rev. Mod. Phys. 22, 221 (1950).

${ }^{13}$ C. Kittle, Phys. Rev. 83, 458 (1951).

${ }^{14}$ L. Chen, X. M. Xiong, H. Meng, P. Lv, and J. X. Zhang, Appl. Phys. Lett. 89, 071916 (2006).

${ }^{15}$ G. V. Lewis, C. R. A. Catlow, and R. E. W. Casselton, J. Am. Ceram. Soc. 68, 555 (1985). 